\title{
A Robust Region Based Level Set Framework for Medical Image Segmentation
}

\author{
Yong Yang \\ School of Information Management, Jiangxi University of Finance and Economics, \\ Nanchang 330013, P.R. China \\ greatyyy765@sohu.com
}

\begin{abstract}
In this paper, we present new developments in the formulation of a new class of level set method for medical image segmentation. In this work, a new speed function of level set framework is proposed. The region statistical information, instead of the conventional image gradient information, is fused into the level set fundamental model to improve the robustness of the segmentation for medical images. The new method has some advantages over classical level set methods especially in the situations where edges are weak and fuzzy. A number of experiments on MR, US and CT images were performed to evaluate the new method. Experimental results are given to illustrate the effectiveness and robustness of the method.
\end{abstract}

\section{Introduction}

Medical image segmentation is a fundamental problem in medical image processing with numerous applications including but not limited to medical image analysis, 3D visualization, etc. It is the process that automates or facilitates the delineation of anatomical structures and other regions of interest (ROI) based on attributes such as their intensity and spatial location. Many classical segmentation techniques have been developed and detailed surveys can be found in references [1]-[3]. However, medical images are often corrupted by noise and sampling artifacts, which cause considerable difficulties when applying classical segmentation techniques, such as edge detection and thresholding. Therefore, sophisticated automated and semi-automated techniques are required.

To address medical image segmentation problem, active contour model [4]-[5] has recently become one of the most studied techniques for medical image segmentation, where an initial contour is deformed towards the boundary of the object to be detected by minimizing an energy function.

The classical active contour model is a parametric deformable model. Parametric active contour models are very popular and are successfully used in medical image segmentation for extracting image contours such as edges by Kass et al. [4]. However, they have some disadvantages. To make the final result relatively to be insensitive to the initial conditions, $\mathrm{Xu}$ and Prince [6] suggested the gradient vector flow (GVF) Snake model. The GVF model is useful when there are boundary concavities. However, the GVF still has topological problems. Chakraborty et al. [7] and Poon et al. [8] 
proposed methods to integrate the parametric snake with region segmentation. It requires a priori knowledge of the interest, and can suffer from topological problems. But the parametric deformable models have difficulty in segmentation of topologically complex structures. To overcome this problem, the level set approach was introduced by Osher and Sethian [9] in 1988, and was created to follow the evolution of Ndimensional curves (interfaces) by observing their curvature. Level sets are designed to handle problems in which the evolving interfaces can develop sharp corners and cusps [10]. Most existing shape modeling techniques require that the topology of the object be known before the shape recovery can commence. However, it is not always possible to specify the topology of an object prior to its recovery. One important concern is topological change resulting from tracking the evolution of curve or surface boundaries through time. During their evolution, interfaces may change connectivity and split, thereby undergoing a topological transformation which is often very difficult to follow using traditional approaches. In the level set approach, the convergence to the final result may be relatively independent of the initial shape, and branches, splits and merges can develop without problems as the front moves. Generally, the method may be applied even when no a priori assumptions about the object's topology are made. Most of the challenges in level set approach resulting from the need to construct an adequate model for the speed function.

In this paper, our main contribution is to define a new speed function model, which is based on the image region statistical information. This region statistical information is then incorporated into the level set method instead of the conventional image gradient information. The paper is organized as follows. The level set approach is briefly reviewed in section 2 and our proposed level set framework is described in section 3 . The experimental results are discussed in section 4. Some conclusions are drawn in section 5 .

\section{Level Set Method Model}

The level set method was devised by Osher and Sethian [9]. The main idea in the level set method is to describe a closed curve $\Gamma$ in the image plane as the zero level set of a higher dimensional function $\phi(x, t)$ in $\mathfrak{R}^{3}$. The value of the $\phi$ at some point $x$ is defined by

$$
\phi(x, t=0)= \pm d
$$

where $d$ is the distance from $x$ to $\Gamma(t=0)$, and the sign in Equation (1) is chosen whether the point $x$ lies outside or inside the initial hypersurface $\Gamma(t=0)$. In this manner, $\Gamma$ is represented by the zero level set $\Gamma(t)=\left\{x \in R^{2} \mid \phi(x, t)=0\right\}$ of the level set function, and the initial function $\phi(x, t=0)$ with the property that $\Gamma(0)=\left\{x \in R^{2} \mid \phi(x, t=0)=0\right\}$. The evolution of $\phi(x, t)$ can be modeled as

$$
\frac{\partial \phi}{\partial t}+F\|\nabla \phi\|=0
$$


with a given $\phi(x, t=0)$. At any instant, the position of $\Gamma$ shall be given as the zero level set of evolving function $\phi$. We must have

$$
\Gamma(t)=\left\{x \in R^{2} \mid \phi(x, t)=0\right\}
$$

The speed function $F$ in equation (2) depends on factors like the image gradient. A common choice for $F$ is

$$
F=P(I)(1-\varepsilon k)
$$

where $0<\mathcal{E}<1$ is a constant, $I$ is the image intensity and $k$ is the curvature, obtained from divergence of the gradient of the normal vector to the front, curvature $k$ can be defined as

$$
k=\frac{\phi_{x x} \phi_{y}{ }^{2}-2 \phi_{x} \phi_{y} \phi_{x y}+\phi_{y y} \phi_{x}^{2}}{\left(\phi_{x}^{2}+\phi_{y}^{2}\right)^{\frac{3}{2}}}
$$

The term $P(I)$ in equation (4) is an image-dependent halting criteria that can be calculated as

$$
P(I)=e^{-\left|\nabla G_{\sigma} * I\right|}
$$

where $\nabla G * I$ denotes the image convolved with a Gaussian smoothing filter whose characteristic width is $\sigma$. This halting criterion allows model to stop on high image gradient by reducing speed function to zero, thus aligning it to the object boundary. The final level set equation for segmentation is given by

$$
\frac{\partial \phi}{\partial t}+P(I)(1-\varepsilon k)\|\nabla \phi\|=0
$$

The above motion equation (7) is a partial differential equation in one higher dimension than the original problem. Given the initial value, it can be solved by means of difference operators in a fixed grid via

$$
\phi_{i j}{ }^{n+1}=\phi_{i j}{ }^{n}-\Delta t \cdot h \cdot\left(\begin{array}{l}
\max \left(F_{i j}, 0\right) \nabla^{+} \\
+\min \left(F_{i j}, 0\right) \nabla^{-}
\end{array}\right)
$$

where $n$ is the iterative time, $h$ is the grid step, $\Delta t$ is the time step, $F_{i j}$ is the speed value of pixel $(i, j), \phi_{i j}{ }^{n}$ is the level value of pixel $(i, j)$ at time $n$ and where

$$
\begin{aligned}
\nabla^{+}= & {\left[\max \left(D_{i j}{ }^{-x} \phi, 0\right)^{2}+\min \left(D_{i j}{ }^{+x} \phi, 0\right)^{2}+\right.} \\
& \left.\max \left(D_{i j}{ }^{-y}, 0\right)^{2}+\min \left(D_{i j}{ }^{+y} \phi, 0\right)^{2}\right]^{\frac{1}{2}}
\end{aligned}
$$




$$
\begin{aligned}
& \nabla^{-}=\left[\max \left(D_{i j}{ }^{+x} \phi, 0\right)^{2}+\min \left(D_{i j}{ }^{-x} \phi, 0\right)^{2}+\right. \\
& \left.\max \left(D_{i j}{ }^{+y}, 0\right)^{2}+\min \left(D_{i j}{ }^{-y} \phi, 0\right)^{2}\right]^{\frac{1}{2}} \\
& D_{i j}{ }^{-x} \phi=\frac{\phi_{i j}{ }^{n}-\phi_{i-1 j}{ }^{n}}{\Delta x} D_{i j}{ }^{+x} \phi=\frac{\phi_{i+1 j}{ }^{n}-\phi_{i j}{ }^{n}}{\Delta x} \\
& D_{i j}{ }^{-y} \phi=\frac{\phi_{i j}{ }^{n}-\phi_{i j-1}{ }^{n}}{\Delta y} D_{i j}{ }^{+y} \phi=\frac{\phi_{i j+1}{ }^{n}-\phi_{i j}{ }^{n}}{\Delta y}
\end{aligned}
$$

This implementation allows the function $\phi$ to automatically follow topological changes and corners during evolution.

\section{Proposed Level Set Method}

In this section, a new speed function is proposed which is based on the image region information since the gradient in the conventional level set approach has no meaning for very noisy and low contrast images. This region information is achieved at a global level by a statistical characterization. The core idea is to utilize the probability density function inside and outside of the structure to be segmented. Here, the original speed function proposed by Malladi [11] is employed for it simplicity as:

$$
F=h_{I}(v-\rho k)
$$

where $v$ represents an external propagation force, $k$ is the local curvature of the front and acts as a regularization term. The weighting $\rho$ expresses the importance given to regularization and the term $h_{I}$ is the data consistency and acts as a stopping criterion at the location of the desired boundaries.

Suppose that the image is partitioned into $N$ pixel, labeled by the integers $1,2, \cdots, N$. In most application, the pixel locations or sites will form a regular square lattice. Further suppose that each pixel variable, $x_{i}, 1 \leq i \leq N$ can take any real value, $x_{i} \in R$. The values of the pixel variables are called intensities and arbitrary shading will be denoted $x=\left\{x_{1}, x_{2}, \cdots, x_{N}\right\}$, hence $x \in R^{N}$. In general, it is not possible to observe $x$ directly, instead the observed image $y$ is a degraded copy of $x$.

$$
y_{i}=x_{i}+\varepsilon_{i}, 1 \leq i \leq N
$$

where $\varepsilon_{i} \sim N\left(0, \sigma^{2}\right)$, and $\varepsilon_{i}$ and $\varepsilon_{j}$ are independent when $i \neq j$. Let us consider the image $I(x, y)$ could be the Gaussian distribution: 


$$
P(Y \mid X)=\frac{1}{\sqrt{2 \pi} \sigma} \exp \left\{-\frac{1}{2 \sigma^{2}} \sum_{i=1}^{N}\left(y_{i}-x_{i}\right)^{2}\right\}
$$

The goal is to segment the image $I(x, y)$ into two "homogeneous" regions separated by the contour. The region inside the object contour, $I_{i n}$, represents the object region. The region outside the contour, $I_{\text {out }}$, corresponds to the background region. We use the same method as in [12] to incorporate region information into level set method. The sign of $v$ determines the direction of the external propagation force:

$$
v=\operatorname{Sign}\left\{a_{i} p\left(I_{\text {in }}\right)-\left(1-a_{i}\right) p\left(I_{\text {out }}\right)\right\}
$$

where $p\left(I_{\text {in }}\right)$ and $p\left(I_{\text {out }}\right)$ denote the likelihood of intensity inside and outside the object, and $a_{i}$ is the prior of a image to be inside the object. The likelihood $p\left(I_{i n}\right)$ of the curve evolves inside the considered region is given by a Gaussian distribution as follows:

$$
p\left(I_{i n}\right) \propto \frac{1}{\sqrt{2 \pi} \sigma_{i n}} \exp \left(-\frac{\left(I(x)-\mu_{i n}\right)^{2}}{2 \sigma^{2}{ }_{i n}}\right)
$$

In the same case, where the curve outside the considered region, the likelihood is described by a translated form of the Gaussian as follows:

$$
p\left(I_{\text {out }}\right) \propto \frac{1}{\sqrt{2 \pi} \sigma_{\text {out }}} \exp \left(-\frac{\left(I(x)-\mu_{\text {out }}\right)^{2}}{2 \sigma_{\text {out }}^{2}}\right)
$$

Set $N_{\text {in }}, N_{\text {out }}$ to be the number of pixels in the image object and background regions. The corresponding parameters $\left(\mu_{i n}, \sigma_{\text {in }}\right)$ and $\left(\mu_{\text {out }}, \sigma_{\text {out }}\right)$ of the image object and background regions are estimated using maximum likelihood (ML) algorithm.

$$
\begin{gathered}
\hat{\mu}_{\text {in }}=\frac{1}{N_{\text {in }}} \sum_{(x, y) \in I_{\text {in }}} I(x, y) \\
\hat{\sigma}_{\text {in }}^{2}=\frac{1}{N_{\text {in }}} \sum_{(x, y) \in I_{\text {in }}} I(x, y)^{2}-\hat{\mu}_{\text {in }}{ }^{2} \\
\hat{\mu}_{\text {out }}=\frac{1}{N_{\text {out }}} \sum_{(x, y) \in I_{\text {out }}} I(x, y) \\
\hat{\sigma}_{\text {out }}^{2}=\frac{1}{N_{\text {out }}} \sum_{(x, y) \in I_{\text {out }}} I(x, y)^{2}-\hat{\mu}_{\text {out }}{ }^{2}
\end{gathered}
$$


As discussed in [13], the maximum likelihood estimation of the target shape can be obtained by maximizing the likelihood.

$$
p(I)=p\left(I_{\text {in }}, I_{\text {out }} \mid I\right)
$$

Based on the bayes rule:

$$
p(I)=\prod_{(x, y) \in I_{\text {in }}} p\left(I_{\text {in }}\right) \prod_{(x, y) \in I_{\text {out }}} p\left(I_{\text {out }}\right)
$$

Since the consistency term $h_{I}$ is proportion to the probability $p(I)$ of the closest point on the current interface, the final expression of the new consistency term $h_{I}$ can be defined as:

$$
h_{I}=\exp \{-k \cdot p(I)\}
$$

Therefore, according to equation (13) then the new speed function can be defined as:

$$
F=h_{I}(v-\rho k)=(v-\rho k) \cdot \exp \{-k \cdot p(I)\}
$$

\section{Experimental Results}

To demonstrate the results of our new speed function for the level set framework, we carried out a series of experiments on different modalities medical images, such as MR, US and CT images. The performance of the proposed method is compared with that of standard level set method.

We first consider segmenting the cerebrospinal fluid (CSF) from a 2D brain MR corpus callosum image with poor contrast. Fig.1 (a) shows the original image, Fig.1 (b) shows the initial curve. The results of the standard level set method and the proposed method are displayed in Fig.1 (c) and (d) respectively. In order to give clear vision about the results, Fig.1 (e) and (f) give the contours corresponding to the segmented regions in Fig.1 (c) and (d). It is observed from the results that with the standard level set method the CSF is under-segmented especially in the middle extended region as show in Fig.1 (e), while with the proposed method the CSF is accurately segmented as shown in Fig.1 (f).

We then test the two methods using a US image. As we know, the US image quality is very poor and the region boundaries seem to be very fuzzy. Fig. 2 shows the results on a US image with both strong and fuzzy region boundaries. Fig.2 (a) shows the original image, Fig.2 (b) shows the initial curve, Fig.2 (c) and (d) give the results of the original level set method and our method, respectively. The segmented contours corresponding to Fig.2 (c) and (d) are illustrated in Fig.2 (e) and (f). Compared to the standard level set method, it is noted that the proposed method can effectively segment the region of interest (ROI) even if the extracting features with fuzzy boundary or with poorly defined edges. 


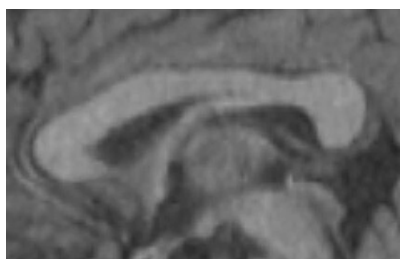

(a)

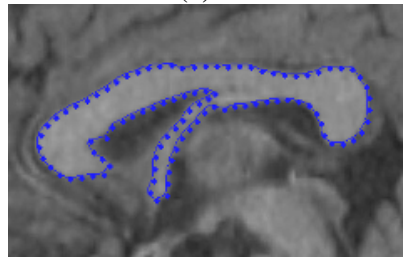

(d)

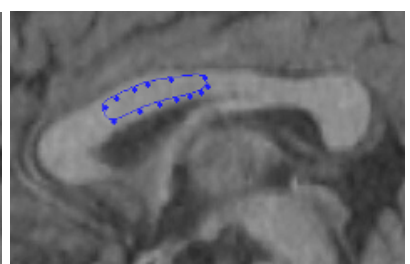

(b)

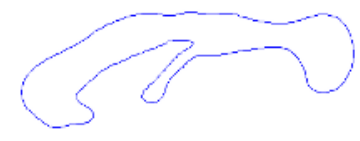

(e)

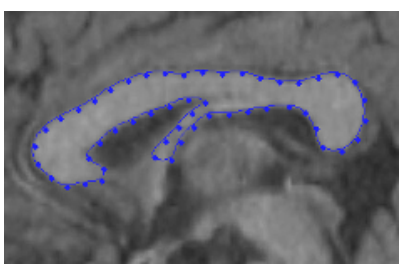

(c)

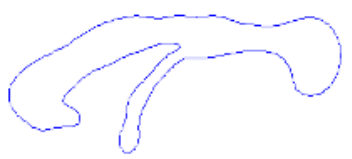

(f)

Fig. 1. Brain MR corpus callosum image segmentation results. (a) Original image; (b) Initial curve; (c) Standard level set method result; (d) Our proposed method result; (e) Contour of the segmented region in (c); (f) Contour of the segmented region in (d).

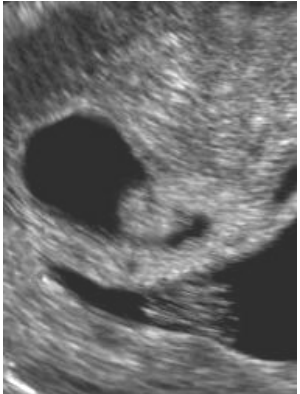

(a)

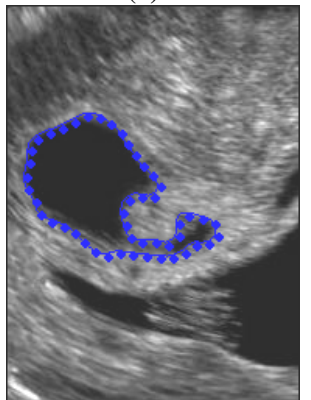

(d)

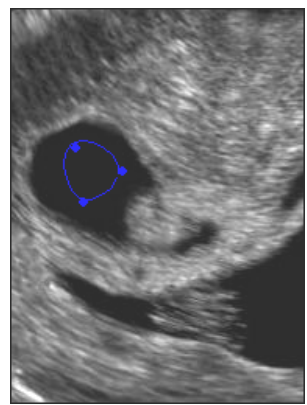

(b)

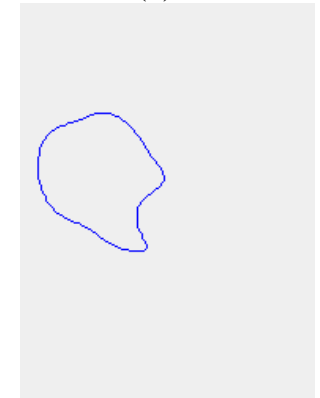

(e)

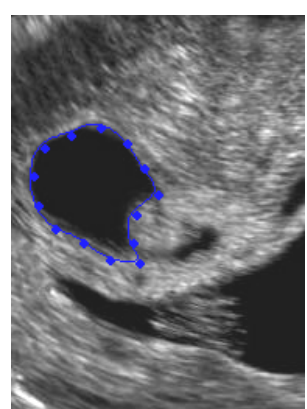

(c)

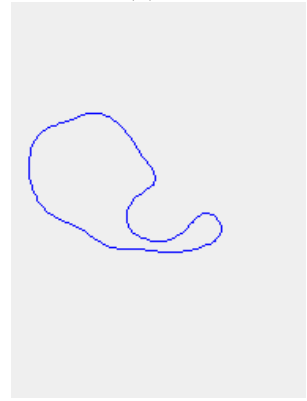

(f)

Fig. 2. US image segmentation results. (a) Original image; (b) Initial curve; (c) Standard level set method result; (d) Our proposed method result; (e) Contour of the segmented region in (c); (f) Contour of the segmented region in (d). 
We also test the robustness of our algorithm to noise. We add Gussian noise to a CT image of the liver as shown in Fig.3 (a), then segment it. Fig.3 (a) is the original image and Fig.3 (b) is the degraded noisy image. The results for comparison are given in Fig.3 (c)-(g). The results clearly demonstrate the superior segmentation quality of our approach in the noisy situation.

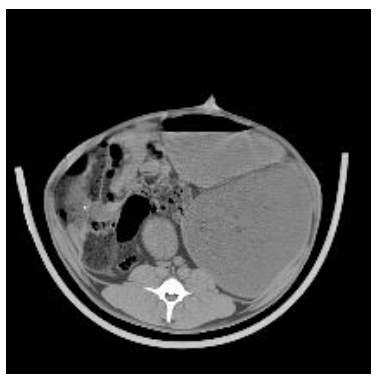

(a)

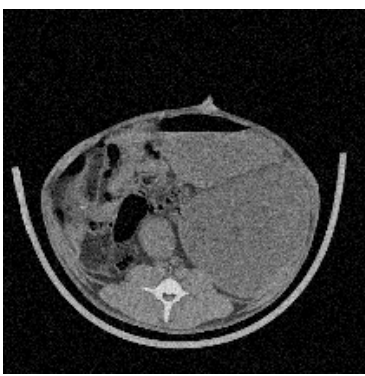

(b)

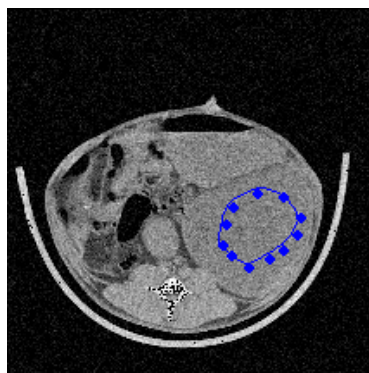

(c)

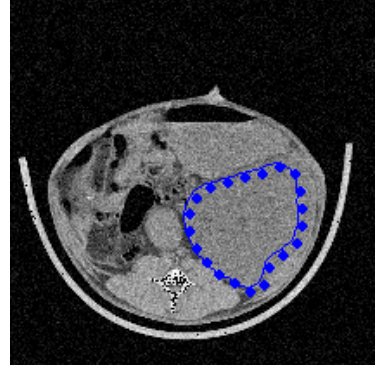

(d)

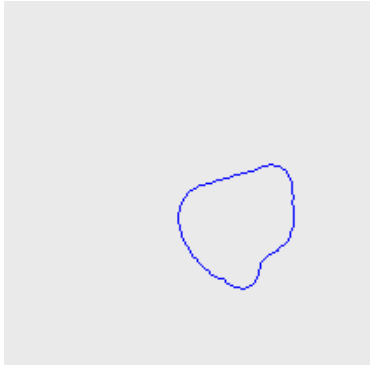

(f)

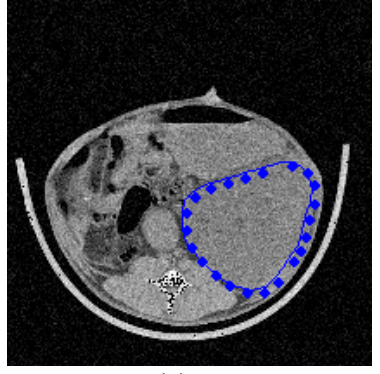

(e)

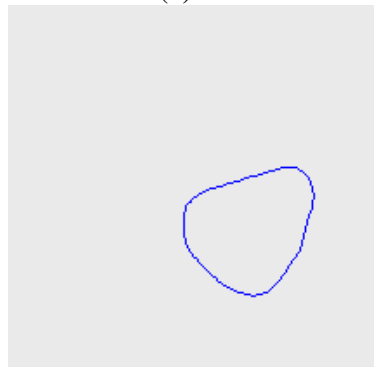

(g)

Fig. 3. CT image segmentation results. (a) Original image; (b) Noisy image; (c) Initial curve; (d) Standard level set method result; (e) Our proposed method result; (f) Contour of the segmented region in (d); (g) Contour of the segmented region in (e).

As mentioned above, we have demonstrated that our method seems ideal for use in a wide variety of medical images. The proposed method can be used to segment images with poor image contrast, fuzzy or diffuse boundary and noise. 


\section{Conclusions}

We have introduced a framework of level set method based on region statistical information. The framework can conveniently incorporate region features into speed function. The method incorporated the region statistical information into the level set fundamental model, instead of the image gradient information. The method has been tested with numerical real modalities medical images, such as MR, US and CT images. We have presented some preliminary experimental results to illustrate the flavor of this technique. The proposed method has been proven to be effective and robust for the segmentation. Future work will focus on the validation of the proposed approach on a variety of medical images.

\section{References}

1. S. K. Fu, J. K. Mui: A survey on image segmentation, Pattern Recognition, Vol. 13, (1981) 3-16.

2. R. M. Haralick, L. G. Shapiro: Image segmentation techniques, Comput.Vision Graphics Image Process., Vol. 29, (1985) 100-132.

3. N. Pal, S. Pal: A review on image segmentation techniques, Pattern Recognition, Vol. 26, (1993) 1277-1294.

4. M. Kass, A. Witkin, and D. Terzopoulos: Snakes: Active contour models, International Journal of Computer Vision, Vol. 1, (1987) 321-331.

5. C. Ciofolo, C. Barillot: Brain Segmentation with Competitive Level Sets and Fuzzy Control., Lecture Notes in Computer Science, Vol. 3565, (2005) 333-344.

6. C. Xu and J. Prince: Generalized Gradient Vector Flow External Forces for Active Contours, Signal Processing, Vol. 71, (1998) 131-139.

7. A. Chakraborty, L. Staib, and J. Duncan: Deformable boundary finding in medical image by integrating gradient and region information, IEEE Trans. Med. Imag., Vol.15, (1996) 859-870.

8. C. S. Poon and M. Braun: Image segmentation by a deformable contour model incorporating region analysis, Phys. Med. Biol., Vol.42, (1997) 1833-1841.

9. S. Osher and J. A. Sethian: Propagating with Curvature Dependent Speed: Algorithms Based on Hamilton-Jacobi Formulations, Journal of Computational Physics, Vol. 79, (1998) 12-49.

10. N. Paragios: A level set approach for shape-driven segmentation and tracking of the left ventricle, IEEE Trans. Med. Imag., Vol. 22, (2003) 773-776.

11. R. Malladi, J. Sethian, and B. Vemuri: Shape modeling with front propagation: A level set approach, IEEE Trans. PAMI, Vol.17, (1995) 158-175.

12. C. Baillard and C. Barillot: Robust 3D segmentation of anatomical structures with level sets, Proceedings of MICCAI, (2000) 237-245.

13. F.Reitich and H.M.Soner: Three-Phase Boundary Motions Under Constant Velocities Part One: The Vanishing Surface Tension Limit, Proc. Royal Soc. Edinburgh, Vol. 126, (1996) 837-865. 